\title{
Optimal Design of Aircraft Landing Gear Mechanism Actuating Force
}

\author{
Chen Huang ${ }^{1}$, Yuhong Jia ${ }^{1}$ \\ 1. School of Aeronautic Science and Engineering, Beihang University \\ Beijing, China \\ huangchen@buaa.edu.cn,jiayuhong@buaa.edu.cn
}

\begin{abstract}
Lowering the actuating force is preferable when designing aircraft landing gear mechanism, which may lessen friction and prevent lock-up of joints. In this research, driving kinematics of operating actuator is modified in addition to geometric dimensions to achieve optimal actuating force during landing gear retraction. The primary purpose is to exploit the dynamics of specific problem and develop optimization strategy with minimum computational cost and compromise in results compared with general approaches. Optimization problem of actuating force is modeled in the context of inverse dynamic analysis of a three-dimensional landing gear mechanism. Actuator brace dimensions and stroke function parameters are chosen as design variables while the maximum power of actuator is limited. Retracting duration and two relative positions of the interpolation point in the stroke function serve as design variables after the optimal dimensions are set. Design of experiments indicates that the ratio between maximum actuating force and maximum actuating power is maximized when the two relative position variables are set equal. Sequential quadratic programming is employed in solving the simplified optimization problem with only two design variables. Lower cost function value is obtained compared with solving the original problem with three driving kinematic variables directly, indicating resistance to converging to local minima in the simplified problem. Global optimization techniques are then used to explore the design space. Results prove that the simplified optimization problem exploiting the dynamics of this problem generates similar optimal actuating force with much smaller computational cost.
\end{abstract}

Keywords—landing gear (aircraft); optimization; dynamics; inverse problems; design of experiments; quadratic programming

\section{INTRODUCTION}

Dynamic analysis is helpful when designing aircraft landing gear mechanism besides a necessary kinematic analysis of landing gear retraction. The actuating force, which can be evaluated by inverse dynamic analysis, is preferred to be as low as possible under certain design constraints. Optimizing actuating force can reduce reaction forces, hence lessen friction and prevent lock-up of joints. It also reduces the required minimum section area of piston, which enables a slender design of actuator that may easily fit into constrained space in some cases.

Traditionally, landing gear retraction is assumed to be quasi-static with an average load factor of 1.25 , hence the actuating force can be estimated by solving static equilibrium equations [1]. With modern analysis tools, it is more convenient to include inertia, friction and aerodynamic force in the dynamic simulation of landing gear retraction to obtain more accurate actuating force. The automation of analysis process also makes design optimization more efficient, which leads to deeper exploration and insights in the design of landing mechanism.

There're several researches on the optimum design of aircraft landing gear mechanism actuating force. Knowles et al. raised a method based on the quasi-static assumption of landing gear retraction and exploits the numerical continuation of constraint equations and static equilibrium equations, which formulates the direct relation between actuating force and geometric dimensions of landing gear mechanism. The response surface of actuating force is generated directly and optimum design variables can be easily found [2 3]. Huang et al. formulated the Euler-Lagrange equation of a threedimensional landing gear mechanism using MATLAB and studied the sensitivity of several geometric parameters, which shows that the position of operating actuator has the dominating effect on the maximum actuating force during landing gear retraction [4]. Chen modeled a landing gear mechanism using ADAMS and studied the effects of gravitational and aerodynamic load on actuating force [5]. Tu et al. studied the effects of actuator dimension by modeling the hydraulic system of landing gear [6]. These aspects can be included in the optimum design of aircraft landing gear mechanism actuating force.

When using geometric dimensions of landing gear mechanism as design variables, design constraints about spatial limitations, kinematic process and structural strength must be defined in the optimization problem, which brings extra complexity and limitations. If the motion of operating actuator is controllable, which can be realized by linear servo motor or hydraulic cylinder with proportional throttle valves [7], the dynamics of landing gear retraction can be further optimized by adjusting the driving kinematics of operating actuator. In this research, a three-dimensional main landing gear mechanism of civil aircraft is modeled and analyzed with given operating actuator stroke function of time. Optimization problem of actuating force is modeled in the context of inverse dynamic analysis of landing gear retraction with actuator brace dimensions and stroke function parameters as design variables. Specific optimization strategy is developed by exploiting the dynamical characteristics of landing gear retraction, which 
reduces computational cost with little compromise in results compared with general global optimization techniques.

\section{INVERSE DYNAMICS OF LANDING GEAR RETRACTION}

A typical three-dimensional landing gear mechanism is shown in Fig. 1. The dynamical model of this mechanism contains 13 rigid bodies, which are the aircraft structure (inertial reference frame), the landing gear strut assembly, cylinder and piston of the main operating actuator, two links of the side stay, two links of the stabilizer stay, cylinder and piston of the auxiliary actuator, and pivots of the three joints at point $\mathrm{F}, \mathrm{G}$, and $\mathrm{H}$. Except for the two universal joints at point $\mathrm{J}$ and $\mathrm{K}$ and two cylindrical joints of two actuators, all other joints are revolute joints.

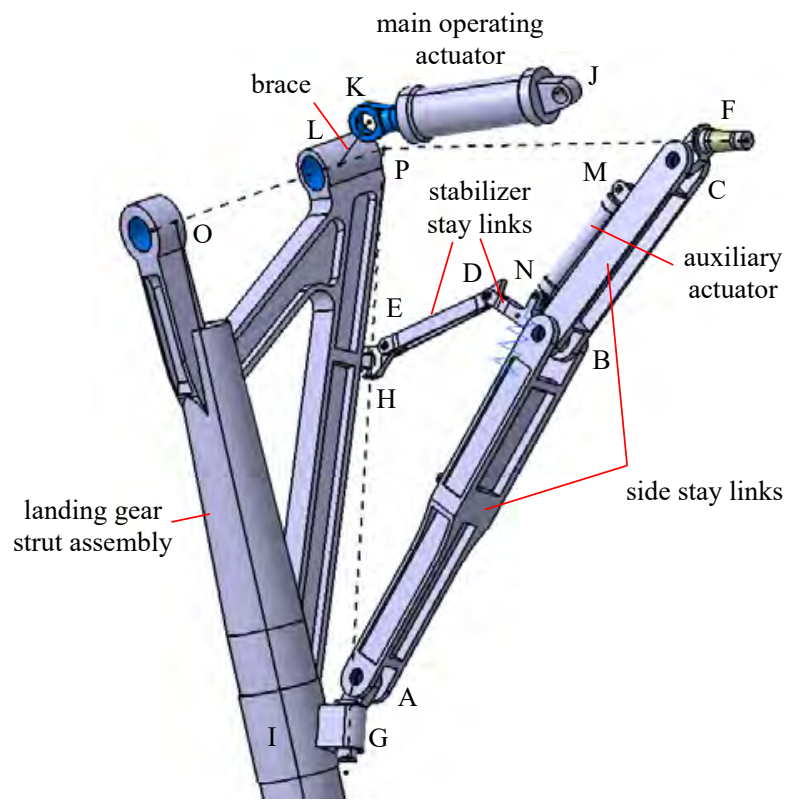

Fig. 1. Three-dimensional main landing gear mechanism

The mechanism has one kinematic degree of freedom after the auxiliary actuator unlocks the stay links. With given main operating actuator stroke function of time $s(t)$ which adds a rheonomous constraint, the mechanism is fully constrained and inverse dynamic analysis can be applied. After removal of redundant constraints by finding the linearly dependent rows of constraint Jacobian matrix, the remaining constraint equations are

$$
\mathbf{C}(\mathbf{q}, t) \equiv\left\{\begin{array}{c}
\mathbf{C}_{\mathbf{j}}(\mathbf{q}) \\
\mathbf{a}_{\mathrm{c}} \cdot\left(\mathbf{r}_{\mathrm{c}}-\mathbf{r}_{\mathrm{p}}\right)+s(t)
\end{array}=\mathbf{0}\right.
$$

where $\mathbf{q}$ are the generalized coordinates of bodies, $\mathbf{C}_{\mathbf{j}}$ are the independent constraints of joints, $\mathbf{a}_{\mathrm{c}}$ is the axial unit vector of operating actuator cylinder, $\mathbf{r}_{c}$ and $\mathbf{r}_{p}$ are the radius vectors of stroke position reference points on actuator cylinder and piston. Starting from a set of known $\mathbf{q}_{1}$ at $t=t_{1}$, the nonlinear constraint equations at $t=t_{2}$ are solved by the least-square iteration $\quad \mathbf{C}_{\mathbf{q}_{i}}^{\mathrm{T}} \mathbf{C}_{\mathbf{q}_{i}}\left(\mathbf{q}_{i+1}-\mathbf{q}_{i}\right)=-\mathbf{C}_{\mathbf{q}_{i}}^{\mathrm{T}} \mathbf{C}\left(\mathbf{q}_{i}, t_{2}\right) \quad$ [8], where the Jacobian matrix $\mathbf{C}_{\mathbf{q}}$ is the partial derivative of $\mathbf{C}$ about $\mathbf{q}$. The time derivatives of (1) are

$$
\begin{gathered}
{\left[\begin{array}{l}
\mathbf{C}_{\mathbf{q}_{j}} \\
\mathbf{C}_{\mathbf{q}_{\mathrm{d}}}
\end{array}\right] \&=-\left\{\begin{array}{l}
\mathbf{0} \\
\&
\end{array}\right\}} \\
{\left[\begin{array}{l}
\mathbf{C}_{\mathbf{q}_{j}} \\
\mathbf{C}_{\mathbf{q}_{\mathrm{d}}}
\end{array}\right]-\mathbf{C}_{\mathbf{q}}^{\&}\left\{\begin{array}{l}
\mathbf{0} \\
\end{array}\right\}}
\end{gathered}
$$

where $\mathbf{C}_{\mathbf{q}_{j}}$ is the submatrix of $\mathbf{C}_{\mathbf{q}}$ about joint constraints and $\mathbf{C}_{\mathbf{q}_{d}}$ is about the actuator driving constraint, are used to solve generalized velocities of and accelerations \&. Then the Lagrange's equations of the first kind

$$
\left[\begin{array}{c}
\mathbf{C}_{\mathbf{q}_{\mathrm{j}}} \\
\mathbf{C}_{\mathbf{q}_{\mathrm{d}}}
\end{array}\right]^{\mathrm{T}}\left\{\begin{array}{l}
\boldsymbol{\lambda}_{\mathrm{j}} \\
F
\end{array}\right\}=\mathbf{Q}-\mathbf{M} \boldsymbol{q}
$$

are solved for joint constraint forces $\lambda_{\mathrm{j}}$ and actuating force $F$. $\mathbf{M}$ is the mass matrix and $\mathbf{Q}$ is the generalized force vector which includes the contributions of gravity, aerodynamic force and joint friction.

The geometric and mechanical model of the landing gear mechanism are built using multibody software Virtual.Lab Motion. Inverse dynamic analysis of landing gear retraction is configured. The important parameters of the dynamic analysis model are listed in TABLE I.

TABLE I. IMPORTANT PARAMETERS OF DYNAMIC ANALYSIS MODEL

\begin{tabular}{|l|l|}
\hline \multicolumn{1}{|c|}{ Parameter name } & \multicolumn{1}{|c|}{ Value } \\
\hline Mass of the strut assembly & $1436.6 \mathrm{~kg}$ \\
\hline $\begin{array}{l}\text { Moment of inertia of the strut assembly along axis } \\
\text { OP }\end{array}$ & $9124.3 \mathrm{~kg} \cdot \mathrm{m}^{2}$ \\
\hline $\begin{array}{l}\text { Rotation angle of the strut assembly during } \\
\text { retraction }\end{array}$ & $80.5^{\circ}$ \\
\hline Maximum stroke of the main operating actuator & $300 \mathrm{~mm}$ \\
\hline Gravitational acceleration & $1.25 \times 9.807 \mathrm{~m} \cdot \mathrm{s}^{-2}$ \\
\hline Maximum aerodynamic drag force & $2334 \mathrm{~N}$ \\
\hline Dynamic coefficient of Coulomb friction & 0.1 \\
\hline
\end{tabular}

With given stroke function that will be detailed in the next section, the actuating force results are shown in Fig. 2 and Fig. 3 . It can be seen that the actuating force generally increases with the stroke position. 


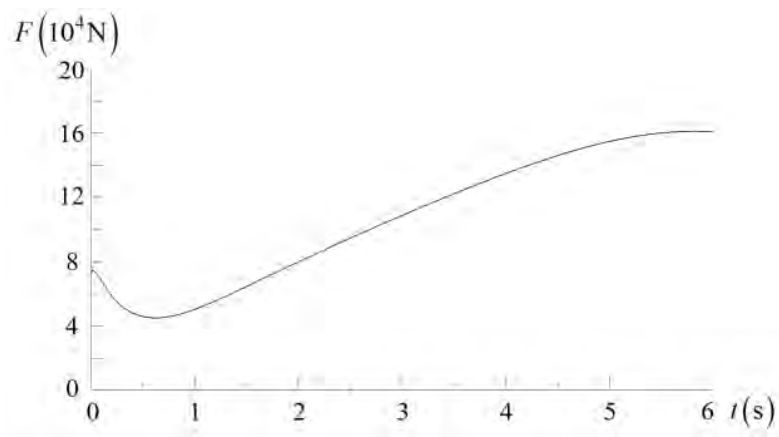

Fig. 2. Actuating force result as a function of time

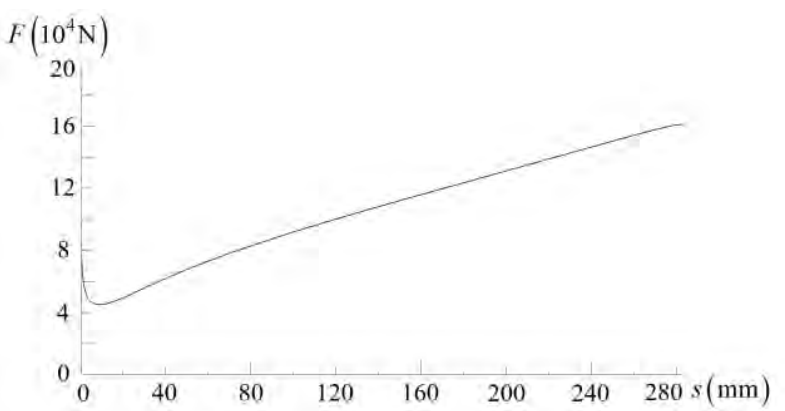

Fig. 3. Actuating force result as a function of actuator stroke position

\section{ACtUAting Force Optimization Problem}

\section{A. Design Variables}

There're two types of design variables in the optimization problem of landing gear mechanism actuating force, which are geometric dimensions and stroke function parameters. Dimensions related to the brace that connecting the strut and main actuator are chosen, including the length of OL, the length of LK, and the angle between LK and plane OPI in Fig. 1 which are denoted as $c, l$ and $a$ respectively. The brace LK is perpendicular to strut rotation axis OP and the positions of point $\mathrm{O}, \mathrm{P}$ and $\mathrm{J}$ are fixed. As the landing gear retraction starts from and ends in static state, the stroke function $s(t)$ should have zero first order derivative at starting and ending time. Also, because the actuating force is varying continuously in real landing gear mechanisms, the accelerations are continuous and should be continuous as well. A cubic spline can approximate the real motion of operating actuator and can be realized by feedback controller ideally. To make the problem simpler, $s(t)$ is designed as a cubic spline that has three interpolation points shown in Fig. 4 and boundary conditions $\&(0)=0$ and $\& T)=0$.

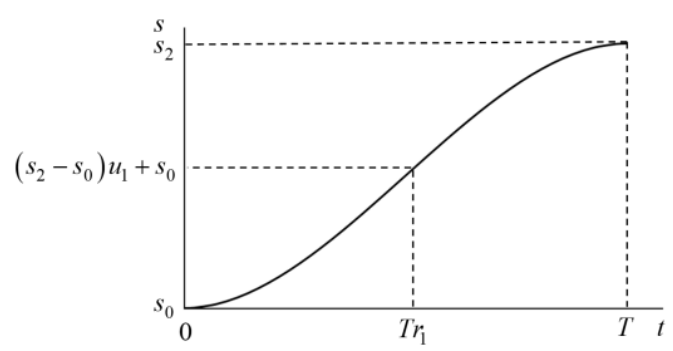

Fig. 4. Operating actuator stroke function as a cubic spline $s_{0}$ and $s_{2}$ are the initial and final stroke positions determined by the geometrics. The target retraction duration $T$ and relative positions of the middle interpolation point $r_{1}$ and $u_{1}$ are the three design variables that define the driving kinematics. The expression of $s(t)$ is

$$
s(t)= \begin{cases}\left(\frac{M_{1}-M_{0}}{6 T r_{1}} t^{3}+\frac{M_{0}}{2} t^{2}+\right. & \\ \left(\frac{s_{1}-s_{0}}{T r_{1}}-\frac{2 M_{0}+M_{1}}{6} T r_{1}\right) t, & 0 \leq t \leq T r_{1} \\ s_{1}+\frac{M_{2}-M_{1}}{6 T\left(1-r_{1}\right)}\left(t-T r_{1}\right)^{3}+\frac{M_{1}}{2}\left(t-T r_{1}\right)^{2}+ & \\ {\left[\frac{s_{2}-s_{1}}{T\left(1-r_{1}\right)}-\frac{2 M_{1}+M_{2}}{6} T\left(1-r_{1}\right)\right]\left(t-T r_{1}\right), T r_{1}<t \leq T}\end{cases}
$$

where parameter $M_{0}, M_{1}$ and $M_{2}$ is the solution of

$$
\left[\begin{array}{ccc}
2 & 1 & 0 \\
r_{1} & 2 & 1-r_{1} \\
0 & 1 & 2
\end{array}\right]\left[\begin{array}{l}
M_{0} \\
M_{1} \\
M_{2}
\end{array}\right]=\frac{6}{T^{2}}\left[\begin{array}{c}
\frac{s_{1}-s_{0}}{r_{1}^{2}} \\
\frac{\left(1-r_{1}\right) s_{0}-s_{1}+r_{1} s_{2}}{r_{1}\left(1-r_{1}\right)} \\
\frac{s_{2}-s_{1}}{\left(1-r_{1}\right)^{2}}
\end{array}\right\rfloor
$$

and $s_{1}=\left(s_{2}-s_{0}\right) u_{1}+s_{0}$.

\section{B. Cost Function}

To minimize the maximum actuating force during landing gear retraction, it serves as cost function directly:

$$
\begin{aligned}
\min f(\mathbf{x}) & =\max \{F(\mathbf{x}, t) \mid 0 \leq t \leq T\} \\
\mathbf{x} & =\left[c, l, a, T, r_{1}, u_{1}\right]
\end{aligned}
$$

It would be extremely complex to obtain the analytical solution of $F(\mathbf{x}, t)$, as well as the gradients of its maximum, hence finite difference must be used for sensitivity analysis and gradient based optimization techniques [9].

\section{Design Constraints}

To ensure the desired motion of operating actuator, the maximum power required must not exceed the power supply limit of hydraulic system:

$$
p(\mathbf{x})=\max \{F \&(\mathbf{x}, t) \mid 0 \leq t \leq T\} \leq p_{\mathrm{c}}
$$


Also, geometric design variables $c, l$ and $a$ should be in certain ranges defined by structural limitations. $T$ should be under the limitation $T_{\mathrm{c}}$ required by aircraft taking off. $r_{1}$ and $u_{1}$ should also be limited to ensure

$$
s_{0} \leq s(t) \leq s_{2}, \forall t \in[0, T]
$$

\section{Optimal Design Scheme}

As the geometric design variables are constrained in relatively small feasible region to ensure proper motion and avoid collisions, the cost function is probably monotonic about these variables, which means that the global optimal design point is on their constraint boundaries. It is safe to study the response about geometric variables separately and determine a set of optimal dimensions, which will effectively simplify the actuating force optimization problem.

Keeping $T=6 \mathrm{~s}$ and $r_{1}=u_{1}=0.5$, cost function response is calculated using equally spaced design of experiments (DOE) in the feasible region of geometric variables shown in Fig. 5.

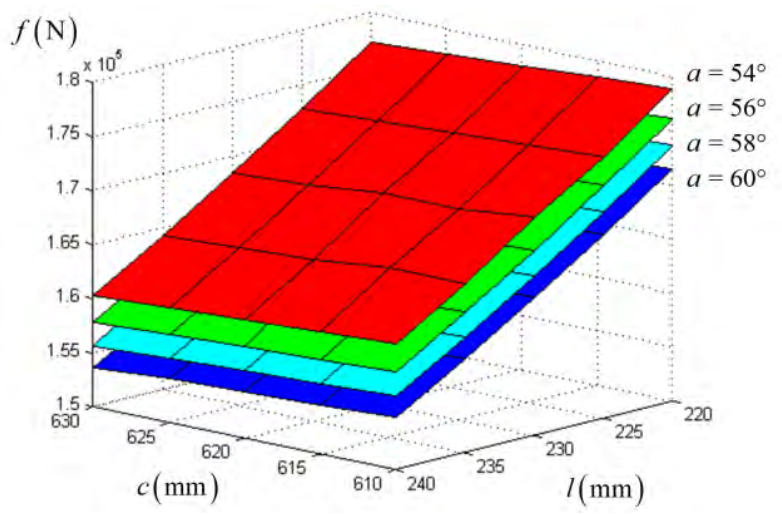

Fig. 5. DOE result of cost function about geometric variables

The maximum actuating force $f$ decreases when $c, l$ or $a$ increases in their allowed ranges, indicating perpendicularity and longer distance between actuator axis $\mathrm{JK}$ and strut rotation axis OP are preferable. The optimal dimension values are $c=630 \mathrm{~mm}, l=240 \mathrm{~mm}$ and $a=60^{\circ}$.

Then the effects of $T, r_{1}$ and $u_{1}$ are studied. As they have relatively wider allowed ranges than geometric dimensions, the maximum power constraint may limit the feasible region. The minimum power required to drive the mechanism would occur when the motion is as slow as possible $\left(T=T_{\mathrm{c}}\right)$, which makes this process quasi-static and the maximum actuating force basically determined by the maximum static load at the end of retraction. The deceleration at the ending phase can balance the gravitational load, hence extra power supply can be used to make the motion faster, increase deceleration and make $f$ lower. If $T$ is set, $r_{1}$ and $u_{1}$ should be adjusted to lower the power requirement. A ratio $\beta(\mathbf{x})=f(\mathbf{x}) / p(\mathbf{x})$ is defined to represent this performance. Setting the geometric variables to previous optimal values and keeping $T=6 \mathrm{~s}$, DOE result of $\beta$ about $r_{1}$ and $u_{1}$ is shown in Fig. 6.

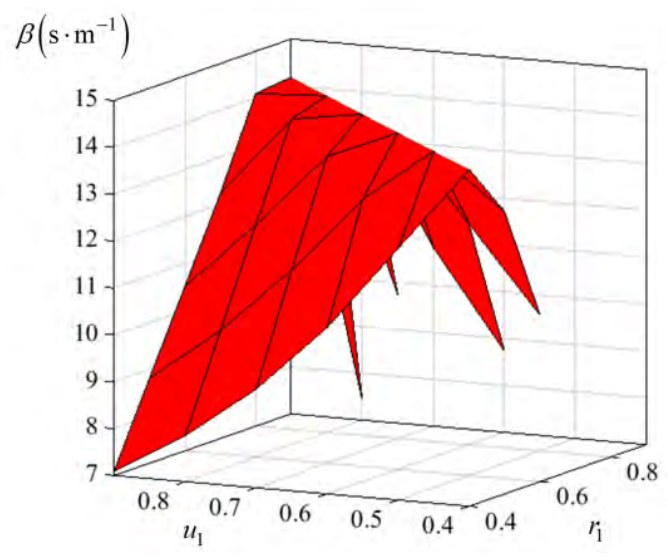

Fig. 6. DOE result of $\beta$ about $r_{1}$ and $u_{1}$

$\beta$ is maximized when $u_{1}=r_{1}$, meaning that lower $p$ is required for certain $t$, which gives more space for shortening $T$ and lowering the maximum actuating force.

Finally, a simplified optimization problem containing only two design variables $T$ and $r_{1}$ is generated with $u_{1}=r_{1}$ and geometric variables set to their optimal values. The cost function is continuous on the feasible region that is also continuous based on the physics of this problem, which makes classic gradient based optimization techniques applicable. The optimization process is modeled using SIMULIA Isight and solved using sequential quadratic programming (SQP). The gradients of $t$ are approximated by their central finite differences [10]:

$$
\nabla f_{k} \approx \frac{f\left(\mathbf{x}+\gamma x_{k}\right)-f\left(\mathbf{x}-\gamma x_{k}\right)}{2 \gamma\left|x_{k}\right|}
$$

where $x_{k}$ is a component of $\mathbf{x}$ and $\gamma=0.01$.

\section{OPTIMIZATION RESULTS COMPARISONS}

Starting from the same initial design point and setting different power limitations, the results of the simplified actuating force optimization problem described in the previous section are given in TABLE II.

TABLE II. RESULTS OF THE SIMPLIFIED PROBLEM SOLVED USING SQP

\begin{tabular}{|l|l|l|l|}
\hline & Initial value & $\boldsymbol{p}_{\mathbf{c}}=\mathbf{1 5 0 0 0 W}$ & $\boldsymbol{p}_{\mathbf{c}}=\mathbf{1 2 0 0 0 W}$ \\
\hline$T(\mathrm{~s})$ & 6 & 4.3754 & 6.2094 \\
\hline$r_{1}$ & 0.5 & 0.7281 & 0.7894 \\
\hline$f(\mathrm{~N})$ & 153820 & $140970(-8.35 \%)$ & $147531(-4.09 \%)$ \\
\hline$p(\mathrm{~W})$ & 11349 & 15000 & 11980 \\
\hline Iterations & & 163 & 101 \\
\hline
\end{tabular}


As a comparison, the optimization problem with three kinematic design variables $T, r_{1}$ and $u_{1}$ is solved from the same initial design point and using the same method and parameters. The results are shown in TABLE III.

TABLE III. RESULTS OF THE THREE DESIGN VARIABLES PROBLEM SOLVED USING SQP

\begin{tabular}{|l|l|l|l|}
\hline & Initial value & $\boldsymbol{p}_{\mathbf{c}}=\mathbf{1 5 0 0 0 W}$ & $\boldsymbol{p}_{\mathbf{c}}=\mathbf{1 2 0 0 0 W}$ \\
\hline$T(\mathrm{~s})$ & 6 & 5.3020 & 6.1024 \\
\hline$r_{1}$ & 0.5 & 0.6902 & 0.7100 \\
\hline$u_{1}$ & 0.5 & 0.5561 & 0.6487 \\
\hline$f(\mathrm{~N})$ & 153820 & $143053(-7.00 \%)$ & $151071(-1.79 \%)$ \\
\hline$p(\mathrm{~W})$ & 11349 & 14991 & 11993 \\
\hline Iterations & & 72 & 89 \\
\hline
\end{tabular}

The problem with more design variables doesn't achieve better results compared with the simplified problem. This indicates that the cost function may not be smooth enough due to numerical errors in dynamic simulation, which cause false local minima. By exploiting the dynamical characteristics of landing gear retraction and setting $u_{1}=r_{1}$, the simplified problem avoids this and enables reliable application of gradient based techniques.

To verify the results, global optimization techniques are employed to solve the three design variables problem. The results of multi-island genetic algorithm (MIGA) and DOE with SQP refinement are given in TABLE IV.

TABLE IV. RESULTS OF THE THREE DESIGN VARIABLES PROBLEM SOLVED USING GLOBAL OPTIMIZATION TECHNIQUES

(a) $p_{\mathrm{c}}=15000 \mathrm{~W}$

\begin{tabular}{|l|l|l|l|}
\hline & \multicolumn{1}{|c|}{ MIGA } & \multicolumn{1}{c|}{ DOE } & SQP refinement \\
\hline$T(\mathrm{~s})$ & 4.3589 & 4.5 & 4.3293 \\
\hline$r_{1}$ & 0.7057 & 0.8 & 0.7867 \\
\hline$u_{1}$ & 0.6832 & 0.65 & 0.6330 \\
\hline$f(\mathrm{~N})$ & $142617(-7.28 \%)$ & $141166(-8.23 \%)$ & $141031(-8.31 \%)$ \\
\hline$p(\mathrm{~W})$ & 14506 & 14913 & 15000 \\
\hline Iterations & 1000 & 1067 & 50 \\
\hline
\end{tabular}

(b) $p_{\mathrm{c}}=12000 \mathrm{~W}$

\begin{tabular}{|l|l|l|l|}
\hline & \multicolumn{1}{|c|}{ MIGA } & \multicolumn{1}{c|}{ DOE } & SQP refinement \\
\hline$T(\mathrm{~s})$ & 6.0745 & 6.5 & 6.4304 \\
\hline$r_{1}$ & 0.7901 & 0.8 & 0.8150 \\
\hline$u_{1}$ & 0.7451 & 0.75 & 0.7611 \\
\hline$f(\mathrm{~N})$ & $147720(-3.97 \%)$ & $148785(-3.27 \%)$ & $147480(-4.12 \%)$ \\
\hline$p(\mathrm{~W})$ & 12000 & 11604 & 12000 \\
\hline Iterations & 1000 & 1067 & 102 \\
\hline
\end{tabular}

The results prove that the simplified optimization problem solved with SQP generates similar results compared with global techniques, while much smaller computational cost is involved.

\section{CONCLUSIONS}

In the optimization problem of aircraft landing gear mechanism actuating force, the driving kinematic parameters can be included as design variables besides geometric dimensions, and their effects can be studied separately. The response of cost function about dimensions of the brace is monotonic, which determines an optimal set of values. Setting two relative positions of the middle interpolation point of main operating actuator stroke function equal will lower the maximum power required for retracting the landing gear, which gives more space for shortening retraction duration and lowering the maximum actuating force. The simplified optimization problem with only two driving kinematic variables is formed and solved using SQP. Lower cost function value is achieved compared with solving the problem with three variables directly using the same method and parameters, indicating resistance to converging to false local minima in the simplified problem. The optimization strategy exploiting the dynamical characteristics of landing gear retraction can reduce computational cost with little compromise in results compared with general global optimization techniques.

\section{REFERENCES}

[1] N.S. Currey, Aircraft Landing Gear Design: Principles and Practices. Reston, VA: American Institute of Aeronautics and Astronautics, 1988, p. 187.

[2] J.A.C. Knowles, B. Krauskopf, and M. Lowenberg, "Numerical continuation applied to landing gear mechanism analysis," Journal of Aircraft, vol. 48, issue 4, 2011, pp. 1254-1262.

[3] J.A.C. Knowles, B. Krauskopf, and M. Lowenberg, "Numerical continuation analysis of a three-dimensional aircraft main landing gear mechanism," Nonlinear Dynamics, vol. 71, issue 1-2, 2013, pp. 331-352.

[4] F.L. Huang, S. Li, and Y.D. Wang, "Study of mechanical efficiency of a civil aircraft's three dimensional landing gear operation mechanism," Journal of Vibration, Measurement and Diagnosis, vol. 33, issue S1, 2013, pp. 188-191.

[5] L. Chen, "Analysis on the kinematic and dynamic performance of landing-gear retraction," College of Aeronautics and Astronautics, Nanjing University of Aeronautics and Astronautics, Nanjing, 2007.

[6] Y. Tu, X. Xiao, and N. Li, "Computer analysis of large-scale aircraft landing gear retraction and extension control system," Journal of Beijing University of Aeronautics and Astronautics, vol. 39, issue 5, 2013, pp. 595-599. 
[7] C. Huang and Y.H. Jia, "Design and simulation of controllable aircraft main landing gear operating actuator," Journal of Beijing University of Aeronautics and Astronautics, vol. 42, issue 1, 2016, pp. 112-119.

[8] J. García de Jalón and E. Bayo, Kinematic and Dynamic Simulation of Multibody System, Second Edition. New York: Springer-Verlag, 2006, pp. 92-93.
[9] D. Bestle and P. Eberhard, "Analyzing and optimizing multi-body systems," Mechanics of Structures and Machines, vol. 20, issue 1, 1992 , pp. 67-92.

[10] R. Fletcher, Practical Methods of Optimization, Second Edition. New York: Wiley-Interscience, 1987. 